\title{
Quasi-Elliptic Microstrip Low-Pass Filters Using an Interdigital DGS Slot
}

\author{
Atallah Balalem, Ali R. Ali, Jan Machac, Senior Member, IEEE, and Abbas Omar, Fellow, IEEE
}

\begin{abstract}
This letter introduces a DGS slot with an interdigital shape. The resonant frequency of the slot can be easily controlled by changing the length of the metal fingers, without changing the area taken by the structure. Using this slot, two quasi-elliptic lowpass filters were designed, fabricated and tested. The filters have a cut-off frequency of about $3 \mathrm{GHz}$.
\end{abstract}

Index Terms-Defected ground structure (DGS), filter, low-pass filters, microstrip filter, transmission zero.

\section{INTRODUCTION}

D EFECTED ground structures (DGS) for microstrip lines have been attracting researchers in recent years. They have been presented in a number of different shapes for filter applications [1], [2]. This technique is suitable for periodic structures, and for both low-pass and band-pass filters, e.g., [3]-[8]. The DGS applied to a microstrip line causes a resonant character of the structure transmission with a resonant frequency controllable by changing the shape and size of the slot.

This letter introduces a DGS in the form of an interdigital slot. The resonant frequency of the structure with this slot can be controlled by adjusting the distance between the metal fingers without changing the area occupied by the slot or the aperture. Two quasi-elliptic low-pass filters based on this slot were designed and fabricated on an $\mathrm{RO} 4003 \mathrm{c}$ substrate $0.831 \mathrm{~mm}$ in thickness and with a relative dielectric constant of 3.38. The resonant behavior of the interdigital DGS used here introduces transmission zeroes to the filter response and consequently improves its stop-band performance.

\section{INTERDIGITAL DGS}

The proposed interdigital DGS slot is shown in Fig. 1. All dimensions in this letter are in $\mathrm{mm}$. The slot is etched in the ground metallization under the microstrip line. This slot has a major advantage in providing tighter capacitive coupling to the line in comparison to known microstrip DGS structures. Moreover, the resonant frequency of the structure can be controlled by changing the distance between the metal fingers. The resonant frequency of the slot can also be modified by changing the

Manuscript received February 7, 2007; revised March 22, 2007. This work was supported by the Czech Technical University, Prague, Czech Republic and by the Czech Ministry of Education, Youth and Sports under the "Research in the Area of Prospective Information and Navigation Technologies" Project MSM 6840770014

A. Balalem, A. R. Ali, and A. Omar are with the Chair of Microwave and Communication Engineering, University of Magdeburg, Magdeburg 39106, Germany (e-mail: atallah.balalem@et.uni-magdeburg.de).

J. Machac is with Faculty of Electrical Engineering, Czech Technical University, Prague, Czech Republic (e-mail: machac@ fel.cvut.cz).

Digital Object Identifier 10.1109/LMWC.2007.901769

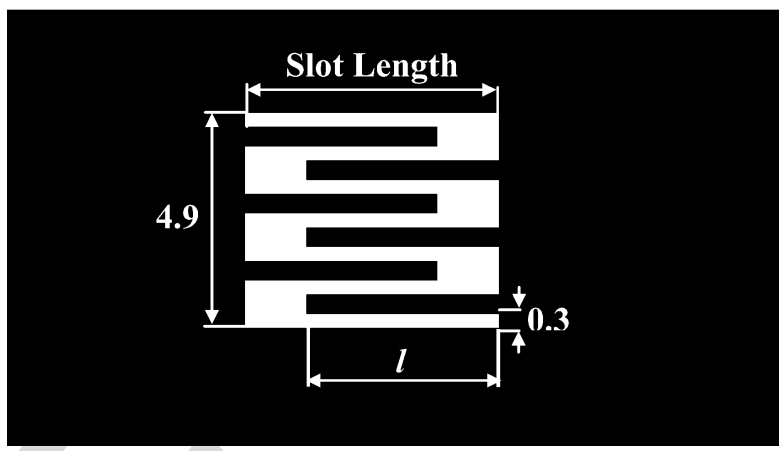

Fig. 1. Bottom side layout of the interdigital DGS structure.

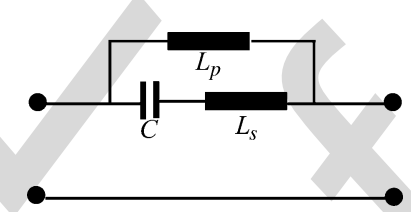

Fig. 2. Equivalent circuit of the proposed interdigital DGS structure.

number of metal fingers, so there is in most cases no need to enlarge the overall slot size.

The equivalent circuit of the proposed structure, illustrated in Fig. 2, is a combination of the interdigital capacitance equivalent circuit [9] and the equivalent circuit of the DGS [1], [2]. The series circuit $C L_{s}$ represents an equivalent circuit of the interdigital capacitance [9].

A parametric study was carried out to show the effect of the finger length on the resonant frequency, particularly on the resonance of the parallel resonator shown in the equivalent circuit in Fig. 2, which represents a transmission zero. This study was done by placing a slot under a transmission line $0.2 \mathrm{~mm}$ wide. The total width of the slot was fixed at $4.9 \mathrm{~mm}$, and the two different slot lengths $S 3.75$ and $5.65 \mathrm{~mm}$ were used. The number of metal fingers was fixed at 6 . The width and spacing of the fingers are equal to $0.3 \mathrm{~mm}$, as shown in Fig. 1. Fig. 3 shows the dependence of the resonant frequency on the length of the metal fingers $l$. By increasing $l$, capacitance $C$ is raised, and therefore the resonant frequency of the slot, the transmission zero, is shifted down. Capacitance $C$ can be as well changed by changing the spacing between the metal fingers. The narrower this spacing is, the higher is the capacitance, and the resonant frequency goes down.

\section{LOW-PASS FILTER WITH AN AdDITIONAL TRANSMISSION ZERO}

In general, the cut-off frequency of the low-pass filter can be adjusted by setting proper values of the lumped elements of the 


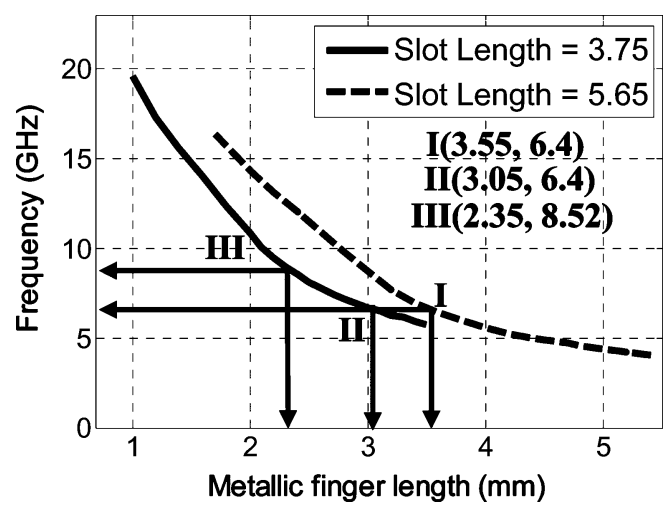

Fig. 3. Parallel resonant frequency of the interdigital DGS structure calculated by SONNET [10] for different lengths of the metallic fingers.

filter [9]. To realize the desired capacitive and inductive values of the filter elements by the stubs of the high/low-impedance transmission lines, the characteristic impedance $Z_{o}$ and effective dielectric constant $\varepsilon_{\text {eff }}$ of these transmission lines have to be determined. For this, a commercial MoM simulator was used [10]. Now the transmission line lengths $l_{k, j}$ of the filter elements, assumed to be much shorter than the wavelength, can be calculated from

$$
\begin{aligned}
C_{k} & =\frac{l_{k}}{Z_{o k} \cdot v_{p h, k}} \\
L_{j} & =\frac{l_{j} \cdot Z_{o j}}{v_{p h, j}}
\end{aligned}
$$

where, indices $k$ and $j$ correspond to stubs with capacitive and inductive character, respectively. $C_{k}$ and $L_{j}$ are the capacitance and inductance of the stubs. The phase velocity

$$
v_{p h}=\frac{c}{\sqrt{\varepsilon_{\mathrm{eff}}}}
$$

where $c$ is the speed of light. Having calculated the transmission line lengths, the filter layout can be constructed, but some additional optimization is still needed.

A microstrip low-pass filter, Fig. 4, was designed on both substrate sides by opening apertures in the ground metallization under the high-impedance transmission line [11]. The aim was to increase the inductance of the narrow strips, which leads to a very compact filter of a small size. However, no resonant effect that can introduce transmission zeroes into the filter response was achieved in this structure. Replacing some of the apertures by the proposed interdigital structure introduces transmission zeroes. The number of transmission zeroes is equal to the number of apertures replaced.

One transmission zero is introduced into the filter response by replacing the central aperture by the interdigital slot structure. This slot, however, shifts the cut-off frequency of the filter down. To shift the cut-off frequency back, it is necessary to reduce the inductance of the narrow stripline that is located over the slot. This can easily be done by increasing the width of the strip. Fig. 5 shows the top and bottom layouts of the designed filter. Fig. 6 shows the measured and simulated return and insertion loss of the filter. A transmission zero, which improves be-

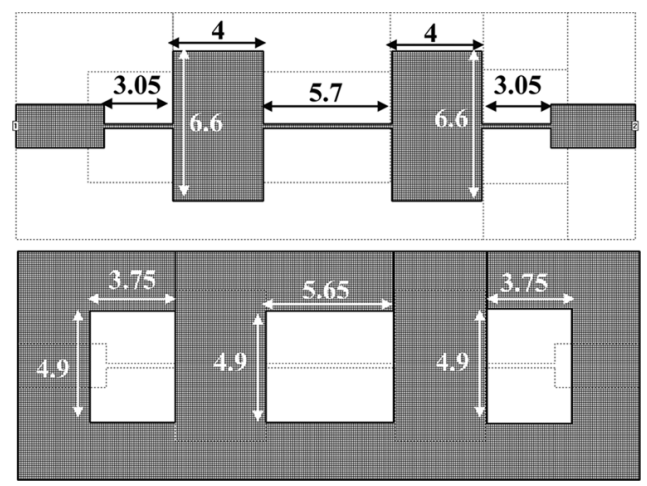

Fig. 4. Top (above) and bottom (below) layouts of a fifth-order low-pass filter with apertures under the high-impedance transmission lines.

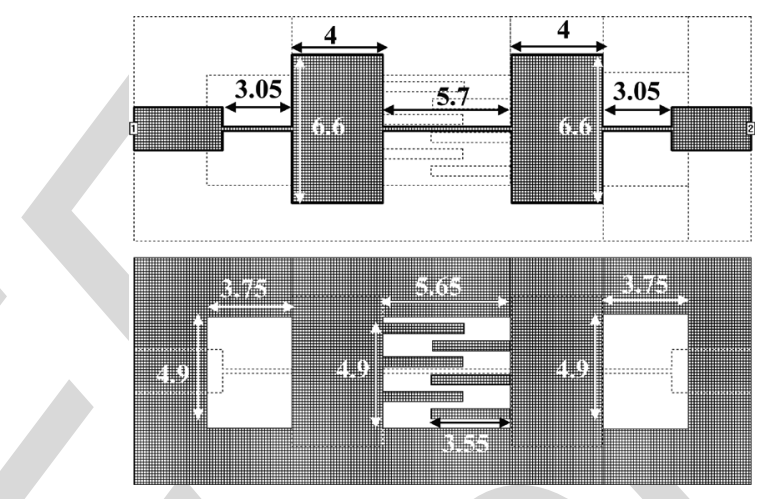

Fig. 5. Top (above) and bottom (below) layouts of a fifth-order low-pass filter with one interdigital DGS slot.

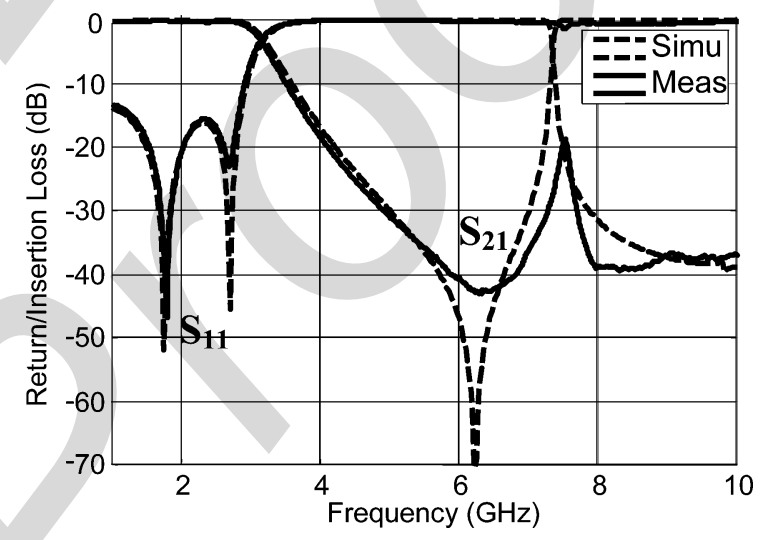

Fig. 6. Return and insertion loss of a fifth-order low-pass filter with one transmission zero.

havior of the filter stop band, is observed at $6.32 \mathrm{GHz}$. This frequency differs slightly from that predicted and marked by point I in Fig. 3 due to the detuning effect of top metal patches. The calculated group delay varies within the pass-band by $0.275 \mathrm{~ns}$.

\section{LOW-PASS Filter With Two AdDitional TRANSMISSION ZEROES}

Two interdigital slots with different finger lengths inserted in the positions of the two apertures introduce two transmission zeroes. Fig. 7 shows the top and bottom layouts of the filter. 


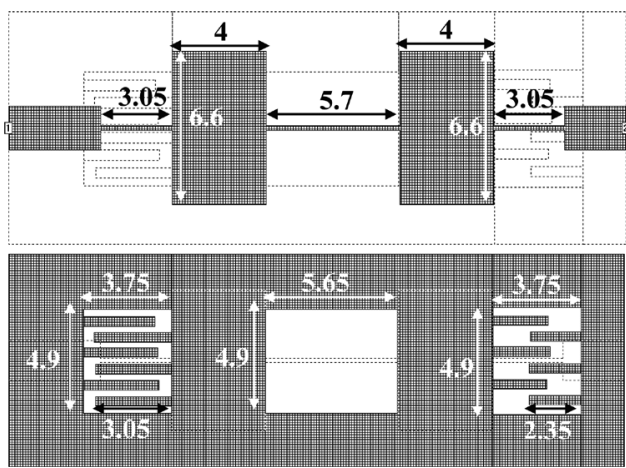

Fig. 7. Top (above) and bottom (below) layouts of a fifth-order low-pass filter with two interdigital DGS slots.

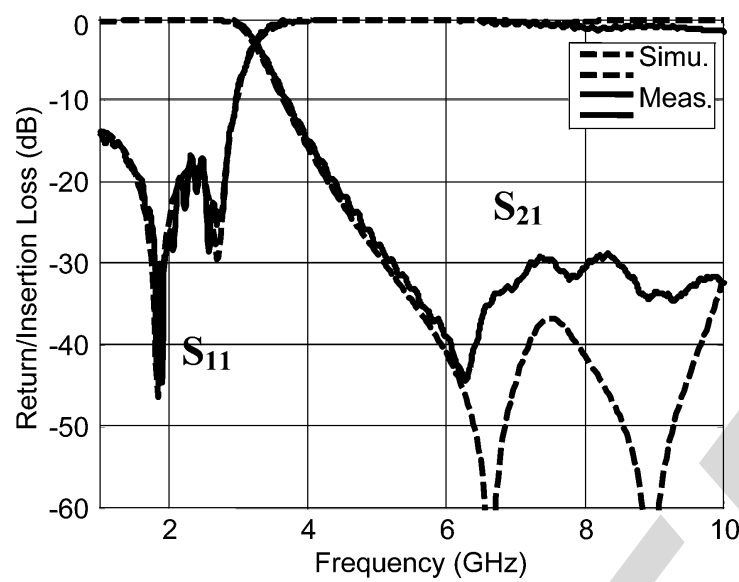

Fig. 8. Return and insertion loss of a fifth-order low-pass filter with two transmission zeroes.

Fig. 8 shows the simulated and measured insertion and return loss of the filter. Two transmission zeros are introduced to the filter response at about 6.45 and $8.6 \mathrm{GHz}$ are slightly different from those calculated and marked by II and III in Fig. 3. Consequently a wide stop-band was achieved. The calculated group delay varies within the pass-band of this filter by about $0.26 \mathrm{~ns}$.

\section{CONCLUSION}

An interdigital DGS slot was introduced in this letter and applied for filter design. The main advantages of the proposed DGS structure are its compact size and the fact that the parallel resonant frequency can be controlled without changing the overall slot area of the DGS. Very compact quasi-elliptic low-pass filters using this DGS were designed and fabricated. The transmission zeros introduced to the filter responses improve their stop-band behavior. Good agreement was achieved between the simulated and measured data.

\section{REFERENCES}

[1] D. Ahn, J.-S. Seok, C.-S. Kim, J. Kim, Y. Qian, and I. Itoh, "A design of low-pass filter using the novel microstrip defected ground structure," IEEE Trans. Microw. Theory Tech., vol. 49, no. 1, pp. 86-93, Jan. 2001.

[2] J. S. Yun, G. Y. Kim, J. S. Park, D. Ahn, K. W. Kang, and J. B. Lim, "A design of the novel coupled line bandpass filter using defected ground structure," in IEEE MTT-S Int. Dig., Boston, MA, Jun. 2000, pp. 327-330.

[3] A. B. Abdel-Rahman, A. K. Verma, A. Boutejdar, and A. S. Omar, "Control of bandstop response of Hi-Lo microstrip low-pass filter using slot in ground plane," IEEE Trans. Microw. Theory Tech., vol. 52, no. 3, pp. 1008-1013, Mar. 2004.

[4] H.-W. Liu, Z.-F. Li, W. Sun, and J.-F. Mao, "An improved 1-D periodic defected ground structure for microstrip line," IEEE Microw. Wireless Compon. Lett., vol. 14, no. 4, pp. 180-182, Apr. 2004.

[5] C.-S. Kim, J.-S Lim, J.-H. Kim, and D. Ahn, "A design of a miniaturized 2-pole bandpass filter by using slot and hair-pin line," in IEEE MTT-S Int. Dig., Fort Worth, TX, Jun. 2006, pp. 1983-1986.

[6] A. R. Ali, A. Abdel-Rahman, A. Amari, and A. S. Omar, "Direct and cross-coupled resonator filters using defected ground structure (DGS) resonators," in Proc. 35th Eur. Microw. Conf., Paris, France, Oct. 2005, pp. $1275-1278$

[7] A. Abdel-Rahman, A. K. Verma, A. Boutejdar, and A. S. Omar, "Compact stub type microstrip bandpass filter using defected ground plane," IEEE Microw. Wireless Compon. Lett., vol. 14, no. 4, pp. 136-138, Apr. 2004.

[8] S.-W. Ting, K.-W. Tam, and R. P. Martins, "Compact microstrip quasi-elliptic bandpass filter using open-loop dumbbell shaped defected ground structure," in IEEE MTT-S Int. Dig., San Francisco, CA, Jun. 2006, pp. 527-530.

[9] G. Matthei, L. Young, and E. M. T. Jones, Microwave Filters, Impedance Matching Networks, and Coupling Structures. Norwood, MA: Artech House, 1980.

[10] "SONNET, Version 10.52. Sonnet Software, Inc., 2007.

[11] L. Zhu, H. Bu, K. Wu, and M. Stubbs, "Unifield CAD model of microstrip line with rear side aperture for multilayer integrated circuits," in IEEE MTT-S Int. Dig., Boston, MA, Jun. 2000, pp. 981-984. 